\title{
Re-irradiation of a second localization of idiopathic midline destructive disease in the head and neck area
}

\author{
Robert L. Poorter $\cdot$ Remco de Bree $\cdot$ C. René Leemans $\cdot$ \\ Paul van der Valk $\cdot$ Ben J. Slotman \\ Johannes A. Langendijk
}

Received: 16 April 2007 / Accepted: 22 June 2007 / Published online: 24 July 2007

(C) Springer-Verlag 2007

\begin{abstract}
Idiopathic midline destructive disease is a rare disease, characterized by a progressive ulceration and destruction of midline facial structures. We report a case with localization on the palate for which she received radiotherapy. Later she developed a second localization on the posterior pharyngeal wall for which she was re-irradiated, without severe sequels. Twice a complete regression was observed.
\end{abstract}

Keywords Idiopathic midline destructive disease . Radiotherapy $\cdot$ Midline granuloma

R. L. Poorter

Department of Radiation Oncology,

Dr. Bernard Verbeeten Institute, Tilburg, The Netherlands

R. de Bree $(\square) \cdot$ C. R. Leemans

Department of Otolaryngology/Head and Neck Surgery,

VU University Medical Center, De Boelelaan 1117,

1081 HV Amsterdam, The Netherlands

e-mail: r.bree@vumc.nl

P. van der Valk

Department of Pathology, VU University Medical Center,

Amsterdam, The Netherlands

B. J. Slotman

Department of Radiation Oncology,

VU University Medical Center, Amsterdam, The Netherlands

\section{J. A. Langendijk}

Department of Radiation Oncology,

University Medical Center Groningen,

Groningen, The Netherlands

R. de Bree

PO Box 7057, 1007 MB Amsterdam, The Netherlands

\section{Introduction}

Idiopathic midline destructive disease is a rare, which is characterized by a progressive ulceration and destruction of midline facial structures, including the nasal cavity, the paranasal sinuses and the palate. In most cases, radiotherapy is an effective treatment [1]. We describe a woman with twice a localization of idiopathic midline destructive disease, which were both treated with irradiation.

\section{Case report}

A woman, 46 years of age, was referred with complaints of nasal discharge while drinking. She was otherwise healthy and there was no history of substance use. Physical examination revealed an almost complete destruction of the membranous nasal septum and perforations of the soft and hard palate of 3 and $5 \mathrm{~mm}$, respectively. Further examination of the head and neck region and general examination showed no abnormalities. Computed tomography showed extensive destruction of the hard palate. Biopsy showed a non-specific inflammation and fibrosis (Fig. 1). There were no signs of Wegener's granulomatosis, malignant lymphoma, tuberculosis or Epstein-Barr virus infection. Serum tests showed negative results for anti-neutrophil cytoplasmic antibodies (ANCA) and syphilis. A diagnosis of idiopathic midline destructive disease was made.

The patient was then treated with radiation therapy to a total dose of 52 Gy in 26 fractions. At the end of the radiation course, a grade 1 mucositis and grade 1 erythema were observed. A complete regression of the disease occurred. After 4 years, the defect in the palate was closed surgically using a temporalis muscle flap. 
Fig. 1 Histological picture of idiopathic midline destructive disease detail $(40 \times$ objective, Hematoxilin \& Eosin stain) showing predominantly small irregular lymphocytes admixed with histiocytes and some granulocytes. The lymphoid cells do not appear obviously malignant. Low power view of biopsy $(5 \times$ objective,

Hematoxilin \& Eosin stain) show an ulcerative surface (left) and the infiltrate (right) with prominent vessels with swollen endothelial cells

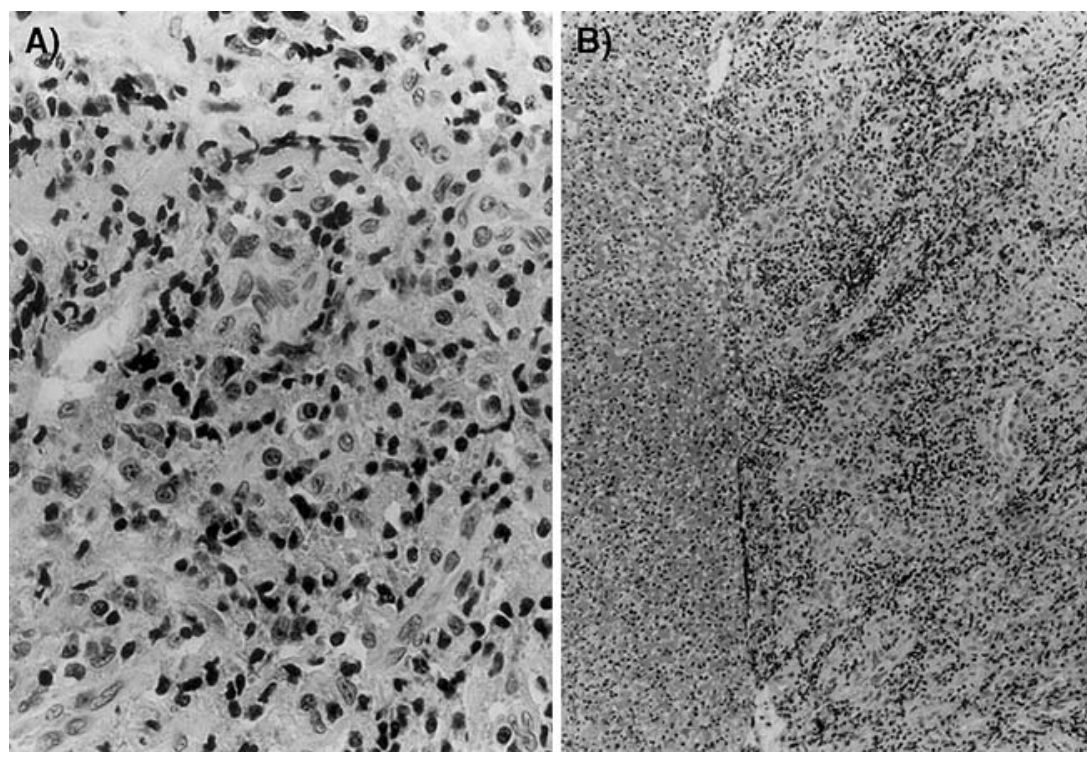

Four years later she developed a sore throat and swallowing complaints. On examination, a tumor-like lesion with a central ulcer was seen on the posterior pharyngeal wall. A CT-scan of the head and neck region showed a soft tissue mass on the posterior pharyngeal wall with extension from the level of the soft palate and nasopharynx to the level of the epiglottis. Biopsy showed the same histologic appearance as previously. The site of the original lesion was without any signs of abnormalities.

It was decided to treat this new localization with re-irradiation. During treatment, the patient experienced grade 1 erythema and grade 2 mucositis. She experienced swallowing complaints for 4 months. Complete regression occurred again.

Four years after re-irradiation the patient presented again with progressive swallowing complaints and nasal speech. On physical examination, an ulcer was seen on the posterior pharyngeal wall with a diameter of $1-\mathrm{cm}$, which had a different clinical appearance than the former two lesions. Therefore, the differential diagnosis included a radiationinduced ulcer or recurrence midline destructive disease. Biopsy was not taken because it was most likely that pathological examination of this lesion could not distinguish between these two entities and because of an increased probability of wound-healing problems after biopsy in a highly irradiated area. We decided to treat this lesion as a radiation ulcer and the patient was proposed to undergo hyperbaric oxygen therapy, which she refused. Therefore, as an alternative, we decided to treat her with pentoxyfilline and high dose vitamin $\mathrm{E}$, which she has used for one year. In the subsequent 12 months, the ulcer as well as her complaints gradually decreased.

During the last follow-up visit (more than 5 years after re-irradiation), there were no signs of recurrent tumor or other severe radiation sequels, while a small ulcer still persists. Besides a mild xerostomia, she had no other complaints.

\section{Discussion}

Idiopathic midline destructive disease (IMDD), also known as lethal midline granuloma (LMG), is a rare disease which is clinically characterized by a progressive, unrelenting ulceration and necrosis of the nasal cavity or the midline facial structures. This disorder has to be distinguished from other progressive, ulcerative disorders of the midline facial tissues, including cocaine use, sarcoidosis, infections like tuberculosis and syphilis, various neoplasms like vestibular or septal carcinoma, Wegener's granulomatosis, and polymorphic reticulosis (PMR) [2]. In contrast to all the other diseases, which have distinct histopathological features that distinguish them from IMDD, the histopathological findings of IMDD are nonspecific. It is essentially a diagnosis per exclusionem.

Some authors believe that IMDD is an early phase of PMR [2]. Others state that all cases referred to as IMDD or PMR are in fact nasal T-cell (natural killer cell) lymphomas (NTL) [1, 3], with a possible association with the EpsteinBarr virus [4]. However, Barker and Hosni recently reported a case of IMDD in which no other cause could be diagnosed by current techniques and they proposed that IMDD remains a valid description in a small number of cases [5]. In our case the clinical course (with only localized disease) and histology (no atypia or Epstein-Barr virus in the tumor) argue strongly against lymphoma and for IMDD.

Untreated IMDD is eventually fatal. No more than approximately 100 cases have been reported, of which only part is characterized as IMDD and the other cases as PMR 
or NTL. Therefore outcome parameters, such as survival and local control, are difficult to interpret. The preferred treatment of IMDD is radiation therapy to a dose of at least 50 Gy $[1,3]$. A 5-year overall survival after radiation therapy has been reported from 15 to $75 \%$. Local control varies between 63 and 77\% [1-4]. Cases reported as NTL do worse compared to IMDD [1]. Some authors administer chemotherapy in case of NTL, although no difference in survival in patients with or without the addition of chemotherapy could be established [3].

In our case, a total dose of $52 \mathrm{~Gy}$ in 26 fractions was used which is generally considered to be sufficient. The margins taken during the first treatment were adequate according to the clinical tumor extension. Also because of the long time-interval, the second lesion can be considered to be a new localization of the disease.

It was decided to treat this new localization with re-irradiation for the following reasons. The largest part of the new localization was outside the initial treatment field implying that only part of the previously irradiated area would be treated again with the second course of radiotherapy. Second, the interval between the initial course of radiotherapy and relapse was more than 8 years and the total dose administered was $52 \mathrm{~Gy}$, which may imply that a substantial amount of initial radiation damage to the normal tissues could have recovered [6-8].

Data on re-treatment of IMDD after local failure are scarce. Both Chen et al. [1] and Sakata et al. [3] mention a case with local relapse with successful re-irradiation without giving any detailed information. One patient with a local relapse of IMDD benefited from salvage chemotherapy [3], whereas two other patients, who were diagnosed as NTL, failed on a combination of re-irradiation and chemotherapy [1]; also on these patients no detailed information regarding radiotherapy was provided.

In conclusion, radiation therapy is the treatment of choice for idiopathic midline destructive disease. In case of a second localization in the irradiated area re-irradiation should be considered.

\section{References}

1. Sakata K, Hareyama M, Ohuchi A, Sido M, Nagakura H, Morita K, Harabuchi Y, Kataura A (1997) Treatment of lethal midline granuloma type nasal T-cell lymphoma. Acta Oncol 36:307-311

2. Perez CA, Chao KSC (1997) Unusual nonepithelial tumors of the head and neck. In: Perez CA, Brady LW (eds) Principles and practice of radiation oncology. Lippincott-Raven, Philadelphia, pp $1108-1110$

3. Chen HH, Fong L, Ting LL, Hong RL, Leung HW, Lui LT (1996) Experience of radiotherapy in lethal midline granuloma with special emphasis on centrofacial T-cell lymphoma: a retrospective analysis covering a 34-year period. Radiother Oncol 38:1-6

4. Hartig G, Montone K, Wasik M, Chalian A, Hayden R (1996) Nasal T-cell lymphoma and the letnal midline granuloma syndrome. Otolaryngol Head Neck Surg 114:653-656

5. Barker THW, Hosni AA (1998) Idiopathic midline destructive disease-does it exist? J Laryngol Otol 112:307-309

6. Eisbruch A, Dawson L (1999) Re-irradiation of head and neck tumors. Hematol Oncol Clin North Am 13:825-836

7. Stevens KR, Britsch A, Moss WT (1994) High-dose reirradiation of head and neck cancer with curative intent. Int J Radiat Oncol Biol Phys 29:687-698

8. Wang CC (1994) To reirradiate or not to reirradiate? Int J Radiat Oncol Biol Phys 29:913 